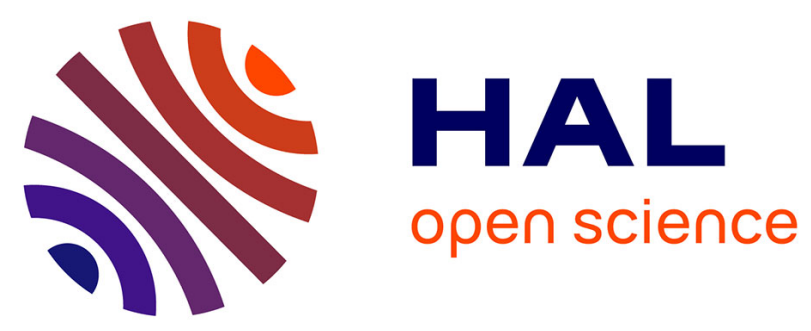

\title{
Quels sont les objets égarés à domicile par les personnes âgées fragiles? Une étude pilote sur 60 personnes
}

\author{
Blandine Boudet, Thérèse Giacobini, Isabelle Ferrané, Carine Fortin,
} Christophe Mollaret, Frédéric Lerasle, Pierre Rumeau

\section{To cite this version:}

Blandine Boudet, Thérèse Giacobini, Isabelle Ferrané, Carine Fortin, Christophe Mollaret, et al.. Quels sont les objets égarés à domicile par les personnes âgées fragiles? Une étude pilote sur 60 personnes. NPG: Neurologie - Psychiatrie - Gériatrie, 2014, 14 (79), pp.38-42. 10.1016/j.npg.2013.10.004 . hal-01138302

\section{HAL Id: hal-01138302 \\ https://hal.science/hal-01138302}

Submitted on 1 Apr 2015

HAL is a multi-disciplinary open access archive for the deposit and dissemination of scientific research documents, whether they are published or not. The documents may come from teaching and research institutions in France or abroad, or from public or private research centers.
L'archive ouverte pluridisciplinaire HAL, est destinée au dépôt et à la diffusion de documents scientifiques de niveau recherche, publiés ou non, émanant des établissements d'enseignement et de recherche français ou étrangers, des laboratoires publics ou privés. 


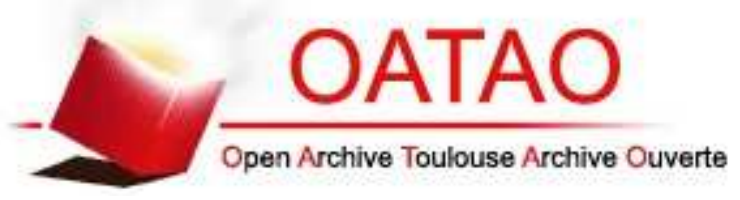

\section{Open Archive TOULOUSE Archive Ouverte (OATAO)}

OATAO is an open access repository that collects the work of Toulouse researchers and makes it freely available over the web where possible.

This is an author-deposited version published in : http://oatao.univ-toulouse.fr/ Eprints ID : 13000

To link to this article : DOI:10.1016/j.npg.2013.10.004

URL : http://dx.doi.org/10.1016/j.npg.2013.10.004

To cite this version : Boudet, Blandine and Giacobini, Thérèse and Ferrané, Isabelle and Fortin, Carine and Mollaret, Christophe and Lerasle, Frédéric and Rumeau, Pierre Quels sont les objets égarés à domicile par les personnes âgées fragiles? Une étude pilote sur 60 personnes. (2014) NPG : Neurologie Psychiatrie - Gériatrie, Vol. 14 ( $\left.{ }^{\circ} 79\right)$. pp. 38-42. ISSN 1627-4830

Any correspondance concerning this service should be sent to the repository administrator: staff-oatao@listes-diff.inp-toulouse.fr 


\title{
Quels sont les objets égarés à domicile par les personnes âgées fragiles? Une étude pilote sur 60 personnes
}

\author{
What are the objects lost by frail elderly at home? A pilot study \\ of 60 people
}

\author{
B. Boudet ${ }^{a, *}$, T. Giacobini ${ }^{a}$, I. Ferrané ${ }^{b}$, C. Fortin ${ }^{c}$, \\ C. Mollaret $^{\mathrm{b}}$, F. Lerasle ${ }^{\mathrm{d}}$, P. Rumeau ${ }^{\mathrm{a}}$ \\ a UMR 1027 Inserm, gérontopôle, laboratoire de gérontechnologie La Grave, CHU Toulouse, \\ université Toulouse-3, place Lange, TSA 60033, 31059 Toulouse cedex 9, France \\ b IRIT UMR 5505, université Paul-Sabatier, 118, route de Narbonne, 31062 Toulouse cedex 9 , \\ France \\ ' ESA (équipe spécialisée Alzheimer) de l'association Alliance SAGES-ADAGES, 1, rue \\ d'Auvergne, 31700 Blagnac, France \\ d LAAS CNRS, 7, avenue du Colonel-Roche, BP 54200, Toulouse cedex 4, France
}

\section{MOTS CLÉS}

Mesures non

médicamenteuses ;

Recherche objets ;

Technologie

d'assistance ;

Troubles mnésiques

\begin{abstract}
Résumé La perte d'objets cause des conflits entre les personnes âgées fragiles et leur famille. Le projet Riddle utilise un robot compagnon pour aider des personnes âgées à retrouver des objets. La bibliographie sur le délire de vol ne donne pas de liste d'objets recherchés. L'objectif est de définir les objets les plus pertinents à localiser en réalisant un interrogatoire croisé, séparément, de 60 couples patient/aidant. Soixante-douze pour cent des patients recherchent des objets ( $82 \%$ pour les aidants). Les objets utilisés les plus recherchés sont : lunettes ( $45 \%)$, clés de maison (34\%), téléphone portable $(31 \%)$, porte-monnaie $(26 \%)$, télécommande $(19 \%)$, canne (22\%). Après équipement technique des objets ainsi définis, la mise en œuvre du service d'aide devra tenir compte de l'usage individuel.
\end{abstract}

Summary Loosing objects is a cause of conflicts between frail elderlies and their caregivers. To our knowledge, the literature addressing delusion of theft doesn't provide information on the objects that are involved. In the RIDDLE project, we are using a companion robot to help the elderly find the objects they are looking for. Hence, we initiated a study with the cross interviews of 60 patient/caregiver dyads to identify which objects would be most relevant to

\section{KEYWORDS}

No drug therapy;

Searching objects; Assistive technology; Memory impairment

* Auteur correspondant. Adresse e-mail : boudet.b@chu-toulouse.fr (B. Boudet). 
com them. Objects are looked for by the patient according to $72 \%$ of the patients and $82 \%$ of the caregivers. The most commonly looked for objects, when they are in use by the patient, are: spectacles (45\%), house keys (34\%), mobile (31\%), wallet $(26 \%)$, remote control $(19 \%)$, and cane $(22 \%)$. After rigging the localization technology to the afore-mentioned objects, the related service will have to be customized to the ways of the user.

Certaines personnes fragiles, ou non, ont tendance à égarer des objets et à accuser leur entourage. Pour l'association France Alzheimer, la perte d'objets fait partie des premiers signes d'alerte de la maladie [1]. Egarer des objets est un symptôme fréquemment rapporté dans la maladie d'Alzheimer [2]. Alzheimer Europe sur son site [3] insiste sur le retentissement de la perte d'objet et le lien social entre le patient et ses aidants avec des accusations de vol influant sur la prise en charge. Une revue de la littérature montre que le délire de vol est un symptôme psychotique rapporté dans un tiers des cas dans la maladie d'Alzheimer [4]. L'une des solutions pour éviter les conflits liés à la perte d'objets serait de faciliter leur recherche. Des outils technologiques pourraient les y aider. Le projet ANR CONTINT Riddle [5] a pour objet de développer une assistance à la recherche d'objets par le biais d'un robot compagnon capable de les localiser à la demande. Pour ce faire, il est nécessaire de savoir quels objets sont le plus souvent cherchés par les patients pour pouvoir résoudre les verrous techniques liés notamment à leur taille, leur forme et leur consistance. L'étude de la bibliographie n'a pas permis d'identifier les objets concernés; nous avons donc décidé d'étudier quels objets étaient perdus en réalisant des entretiens dirigés sur des couples (aidant familial/patient), recrutés dans une population bénéficiant d'une prise en charge gérontologique.

\section{Méthode}

En l'absence de référence bibliographique, un questionnaire pour un entretien dirigé a été élaboré lors d'une séance de brainstorming comportant deux chercheurs en sciences de l'ingénieur spécialistes d'interface homme-machine, un chercheur spécialiste de robotique et de capteurs, deux chercheurs en gérontechnologie appliquée aux troubles cognitifs et deux spécialistes de la maladie d'Alzheimer (ergothérapeute et médecin). La première version du questionnaire a été utilisée sur trois couples tests aidant/patient pour s'assurer qu'il ne dépasse pas 15 minutes par personne. Une version modifiée qui répondait à l'exigence de durée a été réalisée.

L'aidant puis le patient répondent successivement et de façon séparée. L'expérimentateur note les réponses. Ce questionnaire porte sur la présence d'une recherche d'objet, une liste proposant 16 objets du quotidien et une question ouverte sur des objets supplémentaires éventuels.

\section{Population}

L'objectif était de recruter 60 patients âgés de plus de 65 ans, sans trouble cognitif ou avec une atteinte légère
(MMSE compris entre 20 et 30 [6]), accompagnés d'un aidant familial proche (vivant au domicile du patient ou effectuant plus de trois visites par semaine au domicile du patient), dans deux hôpitaux de jour du pôle gériatrie du $\mathrm{CHU}$ de Toulouse ou par l'ESA (équipe spécialisée Alzheimer) de l'association Alliance SAGES-ADAGES. Les données d'analyse secondaire étaient la date de naissance du patient, l'IADL et l'ADL (I'IADL selon Lawton et l'ADL selon Katz sont additionnées pour donner un score sur 14 [7]) et les pathologies. Le critère d'exclusion était l'absence de compréhension du français.

\section{Analyse logique}

Pour l'analyse des réponses à la question «Cherchezvous (cherche-t-il) des objets?», on a distingué quatre groupes (aidant/patient) de réponses différentes: les groupes «oui/oui» et «non/non» (l'aidant et le patient répondent tous les deux de façon concordante à la question); et les groupes «oui/non» et «non/oui» (l'aidant et le patient répondent de façon contradictoire à la question). En absence de réponse spontanée ou dans la liste des objets cités, que ce soit de la part de l'aidant ou du patient, nous considérons que le patient ne cherche pas d'objets à la maison.

Pour l'analyse des réponses à la question «cherchezvous (cherche-t-il) les objets de la liste?»: nous comptons une occurrence lorsqu'au moins une personne du couple (aidant/patient) répond «oui » à la recherche d'un objet de la liste ; ce qui nous permet de déterminer les objets le plus cherchés par les personnes âgées. Nous avons ensuite calculé le pourcentage de recherche de chaque objet rapporté au nombre de personnes disant l'utiliser, que nous avons dénommé «recherche selon l'usage».

Pour l'analyse des réponses spontanées, nous avons comptabilisé une occurrence si un objet est cité spontanément par au moins une personne du couple aidant/patient.

\section{Analyse statistique}

Une analyse statistique a été réalisée à l'aide du logiciel Statview. Un test de Student apparié a été réalisé pour déterminer si le nombre d'objets cités est fonction de l'IADL (conservé ou altéré), si le fait de rechercher ou non des objets est lié au score du patient au MMSE. Un test de corrélation a été réalisé pour déterminer si le type d'objet recherché est fonction du MMSE (normal MMSE > 24 ou altéré MMSE $\leq 24$ ) [8]. 
Tableau 1 Objets les plus cherchés de la liste et en spontané avec concordance des réponses aidant/patient.

\begin{tabular}{|c|c|c|c|}
\hline & \multicolumn{2}{|l|}{ Liste } & \multirow{2}{*}{$\begin{array}{l}\text { Spontané } \\
\text { Nombre occurrences }\end{array}$} \\
\hline & Nombre d'occurrences & $\%$ concordance & \\
\hline Téléphone fixe & 7 & 87 & 3 \\
\hline Téléphone portable & 15 & 78 & 5 \\
\hline Clés maison & 27 & 73 & 14 \\
\hline Clés voiture & 11 & 88 & \\
\hline Télécommande & 21 & 68 & 3 \\
\hline Médicaments & 9 & 80 & \\
\hline Lunettes & 31 & 82 & 19 \\
\hline Étui lunettes & 9 & 65 & \\
\hline Loupe & 4 & 82 & \\
\hline Porte-monnaie & 28 & 60 & 5 \\
\hline Téléalarme & 3 & 88 & \\
\hline Canne & 5 & 93 & \\
\hline Livre & 9 & 77 & 3 \\
\hline Verre & 4 & 90 & \\
\hline Bouteille d'eau & 2 & 95 & \\
\hline Brosse à dent & 1 & 97 & \\
\hline Carte bleue/vitale & Hors liste & & 7 \\
\hline Papiers & Hors liste & & 13 \\
\hline Outils & Hors liste & & 6 \\
\hline Bijou & Hors liste & & 2 \\
\hline $\mathrm{Sac}$ & Hors liste & & 3 \\
\hline
\end{tabular}

\section{Résultats}

Les volontaires ont été recrutés sur la période du 29/10/2012 au 28/02/2013 soit lors d'un passage dans l'un des hôpitaux de jour, soit lors de leur prise en charge par l'ESA. Soixante couples successifs aidant familial/patient ont répondu au questionnaire. Trois couples éligibles n'ont pas souhaité participer, aucun n'a été exclu. La moyenne d'âge des patients est de 81 ans $( \pm 7,03)$ avec un MMSE moyen de $25( \pm 2,9)$ et un IADL moyen de $10,92( \pm 3,62)$. Les diagnostics des patients recrutés sont: aucune pathologie $(2 \%)$, une plainte mnésique $(40 \%)$, des troubles mnésiques ( $18 \%$ ) ou une DTA diagnostiquée ( $40 \%)$. Trente-trois patients ont un MMSE $>24$ et 27 patients ont un MMSE $\leq 24$.

Quatre-vingt-deux pour cent des aidants estiment que la personne aidée cherche des objets à la maison et $72 \%$ des patients déclarent chercher des objets à la maison. Les réponses des couples sont concordantes dans $80 \%$ des cas (40 oui/oui et 8 non/non).

Seulement $13 \%$ des couples déclarent de façon concordante qu'il n'y a pas de recherche d'objets à la maison. Dans trois cas seulement le patient cherche des objets mais cela n'est pas confirmé par l'aidant (non/oui). Neuf aidants déclarent que la personne aidée cherche des objets alors que celle-ci déclare ne pas en chercher (oui/non).

$\mathrm{Si}$ nous considérons les réponses des 60 couples (Tableau 1):

- les objets cherchés le plus spontanément sont les lunettes $(19 / 60)$, les clés $(14 / 60)$ et les papiers $(13 / 60)$, avec une concordance de $42 \%$ entre les réponses des aidants et celles des patients ;

- dans la liste, les objets les plus cherchés sont les lunettes $(31 / 60)$ avec $82 \%$ de concordance entre aidant et patient, le porte-monnaie $(28 / 60)$ avec $60 \%$ de concordance entre aidant et patient, les clés de maison (27/60) avec $73 \%$ de concordance entre aidant et patient et la télécommande $(21 / 60)$ avec $68 \%$ de concordance entre aidant et patient.

Lorsque la personne utilise un objet (Fig. 1), les lunettes sont cherchées dans $45 \%$ des cas, les clés de maison dans $34 \%$, le téléphone portable apparaît et est cherché dans $31 \%$ des cas, le porte-monnaie dans $26 \%$ des cas, la canne dans $22 \%$ des cas. À noter que la télécommande n'est cherchée que dans $19 \%$ des cas. La téléalarme est cherchée dans $19 \%$ des cas pour les aidants mais jamais pour les patients.

Le nombre d'objets cités n'est pas sur notre population fonction de l'IADL totalement conservé $(2,04 \pm 1,55)$ ou altéré $(1,72 \pm 2,06 ; p=0,66)$.

Le MMSE des patients cherchant des objets est plus haut $24,91 \pm 2,94$ contre $24,18 \pm 2,81$ pour ceux qui n'en cherchent pas $(p=0,01)$.

Il n'y a pas de différence dans le type d'objet recherché entre les patients avec un MMSE > 24 et ceux avec un $M M S E \leq 24$, sauf pour le téléphone portable plus cherché par les personnes avec un MMSE $>24(p=0,025)$. Les objets les plus cherchés pour les deux groupes sont les mêmes (lunettes: $p=0,31$; porte-monnaie: $p=0,06$; clés de maison: $p=0,55$; télécommande: $p=0,40$ ). Lorsqu'ils en ont encore l'usage, les patients du groupe avec un MMSE > 24 cherchent plus et de façon significative le téléphone portable ( $45 \%$ contre $13 \% ; p=0,046)$. Par contre, ils semblent chercher plus, sans atteindre le seuil de significativité, les lunettes ( $42 \%$ contre $31 \%$ ), les clés de la maison (36\% contre $31 \%$ ), le porte-monnaie (33\% contre $17 \%)$, les clés de voiture ( $22 \%$ contre $8 \%$ ), l'étui à lunettes ( $20 \%$ contre $16 \%)$. Pour la télécommande ( $20 \%$ contre $19 \%)$, 


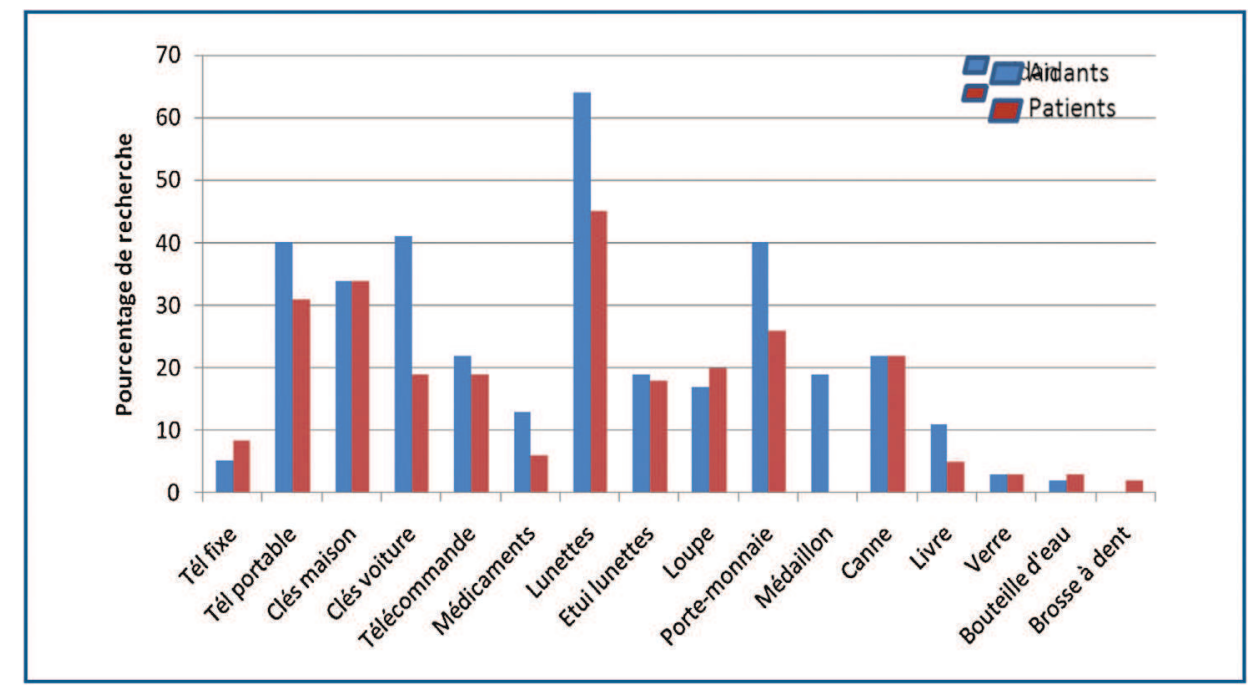

Figure 1. Pourcentage de recherche des objets de la liste (aidant et patient) en fonction de l'usage (objet encore utilisé par le patient).

il n'y a pas de différence visible. La canne, si elle est utilisée, semble, sur des effectifs très faibles, plus souvent recherchée par les personnes ayant un $M M S E \leq 24$ $(25 \%$ contre $17 \%)$. Cette tendance se retrouve dans les réponses spontanées. En effet, les deux groupes cherchent le plus souvent les mêmes objets (lunettes, papiers et clés), mais la prévalence de la recherche est plus importante dans le groupe avec un MMSE $>24$ (lunettes: 12/33 contre $7 / 27, p=0,39$; papiers : $10 / 33$ contre $3 / 27, p=0,07$; clés : $9 / 33$ contre $5 / 27, p=0,43$ ). La téléalarme n'est jamais citée de façon spontanée. Les patients avec un MMSE $\leq 24$ donnent moins de réponses spontanées que ceux avec un MMSE > 24 $(17 / 27$ patients avec MMSE $\leq 24$ contre $14 / 33)$ mais cette différence n'est pas significative $(p=0,11)$.

\section{Discussion}

Les résultats de cette étude montrent que les objets le plus souvent cherchés dans l'absolu par les personnes âgées de plus de 65 ans sont les lunettes, les clés de maison, le porte-monnaie, le téléphone portable, la canne et la télécommande. Pour le développement de la détection par les industriels, il faut prendre en compte ces objets, mais pour la mise en œuvre du service d'aide il faudra choisir les objets pertinents en fonction de l'usage qui en est fait par le patient. Le fournisseur de service devra, pour ce faire, interroger le patient et son entourage. Notre étude n'a pas montré de différence significative dans le fait de chercher des objets en fonction du MMSE ou de l'IADL. Ceci correspondrait à l'absence de corrélation entre le délire de vol et le niveau du MMSE [9]. Néanmoins, l'usage des objets et le niveau de recherche des objets semblent différents en fonction des personnes, de leur atteinte et des objets. En effet, notre étude montre que les personnes avec un MMSE > 24 cherchent plus les objets que celles avec un MMSE compris entre 24 et 20 . Le téléphone portable, qui peut être considéré comme un objet de haute technologie, est significativement plus cherché par les patients les moins atteints, alors que la canne, de basse technologie et d'usage fréquent, est plus cherchée par les patients les plus atteints d'après le MMSE. L'absence de mise en évidence d'un effet statistiquement significatif de l'IADL pourrait être due au fait qu'il s'agissait d'un critère secondaire dans les objectifs de notre étude.

Notre population ne présente que peu ou pas d'atteinte cognitive (MMSE compris entre 20 et 30). Avec des MMSE < 20, la recherche d'objet devrait être moindre si nous tenons compte du fait que l'activité des personnes diminue avec l'aggravation des troubles cognitifs.

Avec une atteinte cognitive faible (MMSE compris entre 20 et 30), la concordance (aidant/patient) des réponses pour chaque objet de la liste est très bonne (>60\%) (Tableau 1 ). On peut supposer que pour des atteintes cognitives plus évoluées, la concordance des réponses entre aidant et patient sera plus faible d'où l'intérêt de faire un interrogatoire croisé.

Notre étude montre une bonne corrélation entre les objets de la liste et les réponses spontanées, en retrouvant globalement les mêmes objets cherchés. Le pourcentage de recherche est globalement plus élevé selon l'aidant que selon le patient (Fig. 1). Cela peut résulter du fait que les patients ne se rendent pas compte qu'ils perdent des objets (agnosie), qu'ils n'acceptent pas leur maladie (déni) ou au contraire que l'aidant majore les symptômes du fait de sa perception (facteur émotionnel amplificateur).

Le choix de débuter par une question ouverte a permis de trouver des objets, non cités dans la liste définie par les experts, mais souvent cherchés comme les papiers et les outils. Il s'agit de classes d'objets avec des caractéristiques variables. Ce résultat n'est pas utilisable pour le développement industriel. La méthode de recueil devra prévoir la demande d'informations complémentaires en cas d'évocation d'une classe d'objets.

Des objets très personnels (bijou, sac) sont cités; ce qui montre la nécessité d'adapter le système de détection des objets à l'individu.

La téléalarme n'est jamais citée de façon spontanée, jamais citée par les patients et peu citée par les aidants, dans la liste comme étant un objet cherché. Ceci correspond à la faible efficacité pratique des téléalarmes, à l'observation quotidienne que l'usage réel des téléalarmes 
est inférieur à leur niveau de mise à disposition. Nous pouvons douter de l'efficacité d'aide à les retrouver pour en promouvoir l'usage.

Il existe des traitements médicamenteux qui semblent diminuer le symptôme psychotique de délire de vol: la rispéridone a été évaluée dans un essai ouvert [10], les autres neuroleptiques, les inhibiteurs de la recapture de la sérotonine ou les anticholinestérasiques sont utilisés empiriquement. Ces traitements ne sont pas dénués d'effets secondaires. La détection des objets grâce à des outils technologiques, ici un robot compagnon (traitement non médicamenteux), pourra aussi aider à limiter les fausses accusations de vol. Ces accusations entraînent une dégradation de la relation entre l'aidant et l'aidé, l'épuisement de l'aidant et l'institutionnalisation [11,12].

\section{Conclusion}

Les aides technologiques peuvent aider les patients et leurs aidants à retrouver les objets. Nous avons défini la liste des objets pertinents à retrouver par notre robot. Une autre équipe (projet ANR TROUVE) [13] travaille sur le principe d'une baguette de sourcier pour retrouver les objets égarés. L'étude des usages permettra de choisir la meilleure solution pour chaque patient dans son environnement.

Il nous paraît que cette étude préliminaire devra être complétée avec un questionnaire affiné au vu des résultats afin de préciser l'effet du niveau d'atteinte cognitive d'une population de patients présentant une maladie d'Alzheimer sur la recherche d'objets.

\section{Déclaration d'intérêts}

Les auteurs déclarent ne pas avoir de conflits d'intérêts en relation avec cet article.

\section{Références}

[1] http://www.francealzheimer.org/sympt\%C3\%B4mes-etdiagnostic/les-premiers-signes-d-alerte/.179

[2] Hamilton L, Fay S, Rockwood K. Misplacing objects in mild to moderate Alzheimer's disease: a descriptive analysis from the VISTA clinical trial. J Neurol Neurosurg Psychiatry 2009;80(9):960-5.

[3] http://www.alzheimer-europe.org/EN/Living-with-dementia/ Caring-for-someone-with-dementia/Changes-in-behaviour / Hiding-losing-objects-and-making-false-accusations\#fragment $-1$

[4] Bassiony MM, Lyketsos CG. Delusions and hallucinations in Alzheimer's disease: review of the brain decade. Psychosomatics 2003; $44: 388-401$.

[5] http://projects.laas.fr/riddle/

[6] Haute Autorité de santé 2011. http://www.has-sante.fr/ portail/upload/docs/application/pdf/2011-12/ recommandation_maladie_d_alzheimer_et_maladies _apparentees_diagnostic_et_prsie_en_charge.pdf

[7] Hugonot-Diener L, Israël L. Activités instrumentales de la vie quotidienne (IADL ou EIADL). In: Hugonot-Diener L, editor. Gremoire. Tests et échelles de la maladie d'Alzheimer et des syndromes apparentés. Marseille: Solal; 2009. p. 229-34.

[8] Folstein MF, Folstein SE, McHugh PR. "Mini-mental state"'. A practical method for grading the cognitive state of patients for the clinician. J Psychiatric Research 1975;12:189-98.

[9] Hwang JP, Yang CH, Tsai SJ, et al. Delusions of theft in dementia of the Alzheimer type: a preliminary report. Alzheimer Dis Assoc Disord 1997;11(2):110-2.

[10] Shigenobu K, Ikeda M, Fukuhara R, et al. Reducing the burden of caring for Alzheimer's disease through the amelioration of "delusions of theft"' by drug therapy. Int J Geriatr Psychiatry 2002; 17:211-7.

[11] Chico GF. Targeting behavior problems in Alzheimer's disease. Emerg Med 2005;37:9-12.

[12] Fischer C, Bozanovic-Sosic R, Norris M. Review of delusions in dementia. Am J Alzheimers Dis Other Demen 2004;19:19-23.

[13] http://www.agence-nationale-recherche.fr/projet-anr/?tx _lwmsuivibilan_pi2 \%5BCODE\%5D=ANR-12-TECS-0005 